\title{
A Comparative Study on the Effects of Occupation Based Reminiscence Therapy and Conversation-Based Reminiscence Therapy Combined with Gymnastics on the Non-demented Elderly in the Community - Randomized Controlled Trial Design
}

\author{
Ju Hyung Park \\ Department of Occupational Therapy, College of Health and Medical Sciences, Cheongju University, Cheongju, South Korea
}

Received December 12, 2021; Revised January 12, 2022; Accepted February 16, 2022

\section{Cite This Paper in the following Citation Styles}

(a): [1] Ju Hyung Park, "A Comparative Study on the Effects of Occupation Based Reminiscence Therapy and Conversation-Based Reminiscence Therapy Combined with Gymnastics on the Non-demented Elderly in the Community - Randomized Controlled Trial Design," Universal Journal of Public Health, Vol. 10, No. 1, pp. 130 - 137, 2022. DOI: 10.13189/ujph.2022.100114.

(b): Ju Hyung Park (2022). A Comparative Study on the Effects of Occupation Based Reminiscence Therapy and Conversation-Based Reminiscence Therapy Combined with Gymnastics on the Non-demented Elderly in the Community - Randomized Controlled Trial Design. Universal Journal of Public Health, 10(1), 130 - 137. DOI: 10.13189/ujph.2022.100114.

Copyright $\bigcirc 2022$ by authors, all rights reserved. Authors agree that this article remains permanently open access under the terms of the Creative Commons Attribution License 4.0 International License

\begin{abstract}
This study is a comparative study examining the effects of conversation-based reminiscence therapy combined with gymnastics and occupation based reminiscence therapy on cognitive function, depression, and quality of life of non-demented elderly in the community. A total of 53 normal elderly subjects in the community were divided into an experimental group $(\mathrm{n}=27)$ and a control group $(\mathrm{n}=26)$. The experimental group received occupation-based reminiscence therapy for 1 hour once a week for a total of 8 weeks, and the control group received 20 minutes of gymnastic exercise and 40 minutes of general conversation-oriented reminiscence therapy. Pre- and post-intervention evaluations of the subjects were performed using the Montreal Cognitive Assessment Korean Version (MoCA-K), a Short Form of the Geriatric depression Scale-Korean Version (SGDS-K), and the Geriatric Quality of Life (GQOL). After the intervention, significant improvements in MoCA-K, SGDS-K, and GQOL were observed in the experimental group $(\mathrm{p}<0.05)$, and significant differences were also observed in comparison with the control group $(p<0.05)$. The results of this study suggest that occupation-based reminiscence
\end{abstract}

therapy can be more effective in cognitive function, depression, and quality of life of the non-demented elderly in the community compared to conversation-based reminiscence therapy combined with gymnastics.

Keywords Dementia, Cognition, Depression, Quality of Life

\section{Introduction}

Dementia is an organic mental disorder and a chronic illness with causes that are generally incurable, and its patients are highly dependent on their families and society [1]. The number of people suffering from dementia is currently estimated as 50 million worldwide, and it is expected to reach 122 million by 2050, a more than three-fold increase [2]. A similar trend is shown in Korea as well. As of 2017, the prevalence of dementia among seniors age 65 and over in Korea was $10.3 \%$, more than 1 in 10 . It is estimated that $15.9 \%$ of the population in Korea 
suffers from dementia in 2050 [2]. As social interest in the prevention of dementia has increased, research and development of various dementia prevention programs targeting the non-demented elderly are being conducted in various fields and domains.

Currently, an effective drug treatment method for preventing dementia has not yet been developed, so non-drug training programs for improving cognitive function, reducing depression, and improving quality of life of the non-disabled elderly are being emphasized $[3,4]$. In a systematic review by Lee et al. of the research related to the development of programs for dementia prevention and cognitive health promotion in old age, the importance of cognitive activity, physical activity, and social activity for reducing the incidence of dementia was mentioned [5]. First, in terms of cognitive activity, this research stated that the more frequent the number of intellectual activities in daily living, the lower the incidence of dementia may be. Next, it was mentioned, in terms of physical activity, when proper physical activity is maintained in daily living, the physical activity helps the brain blood circulation, protects the cranial nerves and strengthens the connectivity between nerve cells, helping to improve overall brain function; in particular, an appropriate level of physical activity performed in earnest before the decline of cognitive function can lead to more positive results in the cognitive function of the subjects. Finally, this research stated that the higher the frequency of social activity of subjects, the slower the rate of decline of cognitive function and the more effective the reduction of the incidence of dementia.

However, the dementia prevention programs that have been introduced in previous studies are individual programs focusing only on subjects' cognitive activity, physical activity, or social activity; furthermore, most of them are mechanical parallel progress forms of two or more programs with different individual focuses, such as sequential performance of gymnastic exercise, cognitive activity and social activity, which means there is still a lack of research on a single program composed of three types of activities including cognitive activity, physical activity, and social activity, integrated at once [6-9].

Reminiscence, which is a mental process of looking back on one's life, is an activity in which one's own past experiences are meaningfully reflected on and told to others [10]. Reminiscence therapy using such nature of reminiscence is an activity in which positive and pleasant contents of one's past experiences are shared with others while talking. It is known that this therapy method, in terms of cognitive activity, not only regenerates one's memory but also provides an opportunity for memory reintegration to improve cognitive function in old age; furthermore, in terms of social activity, it can help through the social interaction with others who share it [11].

In general, such reminiscence therapy can be conducted individually or in groups. Of these approaches, reminiscence therapy implemented in the form of a group, compared to individual reminiscence therapy in the form of one-on-one face-to-face with the subject, promotes interaction with others more effectively, and the individual participants feel a sense of belonging and a sense of security of belonging to a group. Therefore, this therapy method can have the effect of offsetting the psychological difficulties caused by various senses of loss in old age [12, 13].

Occupation based reminiscence therapy, which adds the performance of physical work to this group reminiscence therapy process, involves activities of touching tools and objects that are familiar to subjects or have meaningful experiences or memories in their past together in the group reminiscence therapy process. At the same time, it is a therapy method that allows participants to naturally share experiences and impressions related to the topic, and thus physical activity is added to the existing conversation-centered reminiscence activities as a therapy $[14,15]$. In other words, this occupation-based reminiscence therapy can be regarded as a program that has the advantage of naturally integrating the subjects' physical, cognitive, and social functions owing to the nature of the performance of the activity. However, previous studies of such occupation-based reminiscence therapy have mainly focused on subjects who have already been diagnosed with dementia or mild cognitive impairment (MCI). As such, research on the effect of occupation-based reminiscence therapy on dementia prevention in normal elderly who have not been diagnosed with dementia or mild cognitive impairment (MCI) is still lacking.

Therefore, in this study, conversation-based reminiscence therapy combined with gymnastics and occupation-based reminiscence therapy were applied to non-dementia elderly in the community. The effects of individual treatments on cognitive function, depression, and quality of life of the non-demented elderly were investigated, and their therapeutic effects were compared.

\section{Material and Methods}

This research was conducted targeting normal elderly people over 65 years old who used C Welfare Center located in Cheongju-si, Chungbuk, Korea. The sample size of this research was estimated using $\mathrm{G}^{*}$ Power program 3.1.0 (G power program Version 3.1, Heinrich-Heine-University Düsseldorf, Düsseldorf, Germany). The effect size was set to 0.8 , the significance level $\alpha=0.05$, and the power was set to 0.8 , and the expected sample size required to obtain the minimum statistical power was 26 per group, 52 in total.

The criteria for selecting subjects for this research were determined as normal elderly aged 65 years or older who did not have any cognitive function problems and had a score of 23 points or higher in the Montreal Cognitive Assessment Korean Version (MoCA-K), who were confirmed not to have any neurological disease as the results of examinations within the last 3 months, who did 
not participate in any health-related programs according to a prior interview, and who had no experience in participating in a dementia prevention program at all. In addition, those who were diagnosed with dementia or mild cognitive impairment (MCI), or who had neurological and physical abnormalities such as brain tumors or traumatic brain damage or epilepsy, were excluded from this research.

In this research, 33 subjects per group, a total of 66 subjects, were initially recruited according to the selection and exclusion criteria considering the dropout rate of about $20 \%$ during the research process, but 13 people who expressed their intent not to participate in the program due to personal circumstances during the research process were excluded and thus a total of 53 subjects participated until the final stage of this research. The purpose of this research and the entire process were fully explained to all subjects during the initial recruitment, and the entire research process was conducted after receiving subjects' voluntary written consent. This research passed a review by the Research Ethics Review Committee of Cheongju University (1041107-202102-HR-074-01).

At the beginning of the research, selected subjects were randomly divided into two groups. Sealed envelopes containing cards marked 1 or 2 were used for randomization, with the subjects selected as 1 assigned to the experimental group and the subjects selected as 2 assigned to the control group. Programs given to the experimental group and control group consisted of a total of 10 sessions, including 2 pre-post evaluations and 8 therapy programs. The individual therapy program was conducted once a week, for 1 hour per session. To support the smooth progress of the overall program, three additional assistant hosts participated in all program operations. None of the subjects participated in any program other than the program provided in this research during the research period.

The experimental group $(n=27)$ received occupation-based reminiscence therapy, and the control group $(n=26)$ received gymnastics for physical activity and general conversation-oriented reminiscence therapy. The themes of the occupation-based reminiscence therapy program applied to the experimental group and the general reminiscence therapy applied to the control group were limited to 8 themes on which all subjects had prior experience and had responded to positively in the prior interview before program implementation, among the themes used in the occupation based reminiscence therapy precedent research targeting the elderly with dementia or mild cognitive impairment (MCI) [14-17].

Total subjects screened $(n=66)$

Included for the this research $(\mathrm{n}=53)$

- declined to participate $(\mathrm{n}=13)$

Informed consent taken $(\mathrm{n}=53)$

Baseline Measurement $(\mathrm{n}=53)$

\begin{tabular}{|c|c|}
\hline \multicolumn{2}{|c|}{ Randomized ( $\mathrm{n}=53$ ) } \\
\hline $\begin{array}{l}\text { Experimental group }(n=27) \\
\text { - occupation-based reminiscence therapy } \\
\text { - } 27 \text { subjects completed the trial }\end{array}$ & $\begin{array}{l}\text { Control group }(\mathrm{n}=26) \\
\text { - gymnastics for physical activity and general } \\
\text { conversation-oriented reminiscence therapy } \\
\text { - } 26 \text { subjects completed the trial }\end{array}$ \\
\hline Post- & urement $(\mathrm{n}=53)$ \\
\hline
\end{tabular}

Figure 1. Flow Chart of this research 
The selected activities were three activities of making traditional Korean food (songpyeon, dumplings, and bibimbap), and planting plants in pots, making a simple hand fan, and three activities of making Korean folk toys (kites, yut-nori boards, and tops) (Table 1). Each occupation-based reminiscence therapy program was conducted in three stages for a total of 1 hour. In the introduction stage, the research director prepared photos or production tools related to the work activity to induce the subjects' interest, and in the main stage, conversation among the subjects was encouraged so that the subjects' individual past experiences related to the performance of the work could be shared. In the final stage, subjects were encouraged to share their experiences and impressions of the task by looking at the finished product. The entire program was operated following the operation and guidance of the the research director, and the three assistant hosts of this program provided direct help and demonstrations to individual subjects who lacked understanding or needed help in the context of work activities. In addition, to facilitate reminiscence activities according to the situation, subjects were helped to actively participate in reminiscence and share stories through activities such as asking questions about work tasks that could arouse interest.

Table 1. Activities selected for this study

\begin{tabular}{|c|c|c|}
\hline \multicolumn{3}{|c|}{ Activity name and description } \\
\hline 1 & songpyeon making & \multirow{3}{*}{$\begin{array}{l}\text { Simple Korean traditional food } \\
\text { making activities }\end{array}$} \\
\hline 2 & dumplings making & \\
\hline 3 & bibimbap making & \\
\hline 4 & planting plants in pots & Simple gardening activity \\
\hline 5 & $\begin{array}{l}\text { making a simple hand } \\
\text { fan }\end{array}$ & $\begin{array}{c}\text { Making simple Korean traditional } \\
\text { living tools }\end{array}$ \\
\hline 6 & making a kite & \multirow{3}{*}{$\begin{array}{c}\text { Making a simple Korean folk } \\
\text { toys tool }\end{array}$} \\
\hline 7 & $\begin{array}{l}\text { making a yut-nori } \\
\text { boards }\end{array}$ & \\
\hline 8 & making a tops & \\
\hline
\end{tabular}

For interventions applied to the control group, subjects received general conversation-oriented reminiscence therapy in which the participants would share their memories through a conversation while looking at pictures of the same subject as the tasks applied to the gymnastic exercise and experimental group for physical activity. The gymnastics time was 20 minutes, and the general conversation-oriented reminiscence therapy program was conducted for 40 minutes. The gymnastics activities were conducted through the supervision of the research director and the demonstration and individual guidance of 3 assistant hosts. Health gymnastics and finger gymnastics for muscle strengthening and fall prevention used in previous research on existing Dementia Prevention Programs were used [16]. For general conversation-oriented reminiscence therapy used in the control group, in the introduction stage of the experimental group, the research director prepared photos related to the themes to induce interest in the subjects. In the main stage, subjects were encouraged to share naturally among themselves while communicating on their individual past experiences related to the theme. In the final stage, the participants were encouraged to share their experiences and impressions of the reminiscence therapy. In the conversation-oriented reminiscence activities of the control group, the three assistant hosts asked questions about individual subjects or encouraged them to engage in active dialogue for smooth reminiscence activities.

The subjects were evaluated using the a) Montreal Cognitive Assessment Korean Version (MoCA-K) for cognitive function, b) Short Form of Geriatric Depression Scale-Korean Version (SGDS-K) for severity of depression level, and c) the Geriatric Quality of Life-Dementia (GQOL-D) targeting demented elderly subjects for quality of life. Each evaluation was performed once before and after the intervention activity.

MoCA-K is a Korean version of MoCA, a tool designed by Nasreddine et al. to screen for mild cognitive impairment (MCI), and is an assessment tool whose reliability and validity have been verified through Korean translation and correction to supplement it to suit Korean conditions [18]. This tool was used to investigate changes in the cognitive function of experimental subjects before and after intervention. The specific evaluation items consist of spatiotemporal/executive ability, vocabulary, attentiveness, linguistic ability, abstraction, reminiscence, and orientation. As a screening criterion for mild cognitive impairment (MCI), an evaluation score of 23 or more is considered to be normal, while a score of 22 or less is considered to be mild cognitive impairment (MCI). The highest score of the assessment tool is 30 , and Cronbach's $\alpha=0.81 \sim 0.84$ for this tool [19].

This research used the Short Form Geriatric Depression Scale-Korean Version: SGDS-K, a tool for measuring the severity of depression level for the elderly (19), to investigate the severity of depression level in the subjects. The SGDS-K assigns a score of up to 15 points, and a higher score means a higher level of depression in the subject. Cronbach's a $=0.88$ in this tool was reported, and the test-retest reliability was 0.75 [20].

The quality of life of subjects in this research was measured using GQOL. The detailed items of the GQOL are divided into a total of four areas: physical health, psychological health, social relations and economic level, and physical environment, and it consists of 25 items in total by adding 2 items measuring health and overall life satisfaction [21]. For all items, the highest score is 4 points, and the higher the total score, the higher the subjects' quality of life. The internal consistency of the tool was 0.91 [22].

Data obtained through this research were analyzed using SPSS 23.0 for Windows (IBM Corporation, Armonk, NY, 
USA). To understand the general characteristics of subjects in the experimental group and control group before intervention, and to compare cognitive function, severity of depression level, and quality of life between the groups before intervention, an independent sample T-test and chi-square test were used for analysis. The paired-sample T-test was used to compare each group's cognitive function, severity of depression level, and quality of life before and after the intervention, and the comparison between groups after the intervention was analyzed using the independent sample T-test. The significance level of the analysis was set to 0.05 .

\section{Results}

The general characteristics of the subjects who participated in this research are shown in Table 1. There were no significant differences between the experimental group and the control group in terms of gender, age, and years of education (Table 2).
Table 2 shows the change patterns before and after intervention for each group in the evaluation of cognitive function, severity of depression level, and quality of life, and a comparison between two groups before and after treatment. There were no significant differences between the two groups in cognitive function, severity of depression level, and quality of life before the intervention. In the comparison before and after the intervention for each group, there were no significant differences in cognitive function, severity of depression level, and quality of life in the control group, but a significant difference was found in all evaluations in the experimental group $(\mathrm{p}<0.05)$. In the comparison between the experimental group and the control group before the intervention, there were no significant differences in cognitive function, severity of depression level, and quality of life. However, in the comparison between the experimental group and the control group after the intervention, significant differences were found in all items of cognitive function, severity of depression level, and quality of life $(\mathrm{p}<0.05)$ (Table 3$)$.

Table 2. General characteristics of subjects $(n=53)$

\begin{tabular}{|c|c|c|c|c|}
\hline \multicolumn{2}{|c|}{ Characteristics } & $\begin{array}{l}\text { Experimental group } \\
(n=27)\end{array}$ & $\begin{array}{c}\text { Control group } \\
(\mathrm{n}=\mathbf{2 6})\end{array}$ & $\mathbf{x} 2 / \mathrm{p}$ \\
\hline \multirow{2}{*}{ Gender } & Male & 14 & 13 & \multirow{2}{*}{.893} \\
\hline & Female & 13 & 13 & \\
\hline \multicolumn{2}{|c|}{ Age(years) } & $68.59 \pm 2.678$ & $69.27 \pm 2.491$ & .346 \\
\hline \multicolumn{2}{|c|}{ Education(yr) } & $10.96 \pm 2.084$ & $10.50 \pm 2.285$ & .444 \\
\hline
\end{tabular}

$* \mathrm{p}<0.05$

Table 3. Comparison of change in characteristics of the experimental group and control group with values presented as the mean \pm standard deviation $(\mathrm{n}=53)$

\begin{tabular}{|c|c|c|c|c|}
\hline & & $\begin{array}{l}\text { Experimental group } \\
\qquad(\mathrm{n}=27)\end{array}$ & $\begin{array}{l}\text { Control group } \\
(n=26)\end{array}$ & $\mathbf{t}$ \\
\hline \multirow{3}{*}{ MoCA-K } & pre-test & $25.48 . \pm 1.397$ & $25.85 \pm 1.488$ & -.920 \\
\hline & post-test & $26.85 \pm 1.562$ & $25.96 \pm 1.562$ & $2.075^{*}$ \\
\hline & $\mathrm{t}$ & $-11.315^{*}$ & -1.140 & \\
\hline \multirow{3}{*}{ SGDS-K } & pre-test & $5.85 \pm 1.406$ & $6.04 \pm 1.800$ & -.422 \\
\hline & post-test & $4.41 \pm 2.062$ & $5.92 \pm 2.189$ & $-2.595^{*}$ \\
\hline & $\mathrm{t}$ & $4.618^{*}$ & .293 & \\
\hline \multirow{3}{*}{ GQOL-D } & pre-test & $67.22 \pm 4.371$ & $66.54 \pm 4.868$ & .538 \\
\hline & post-test & $71.07 \pm 6.639$ & $67.12 \pm 1.383$ & $2.105^{*}$ \\
\hline & $\mathrm{t}$ & $-3.196^{*}$ & -.385 & \\
\hline
\end{tabular}

MoCA-K - the Montreal Cognitive Assessment Korean Version; SGDS-K - Short Form of Geriatric Depression Scale-Korean Version; GQOL-D - the Geriatric Quality of Life-Dementia

$* \mathrm{p}<0.05$ 


\section{Discussion}

In this research, occupation-based reminiscence therapy was provided to non-demented normal elderly living in the local community, and the effects on cognitive function, depression level, and quality of life of the subjects was investigated to verify the effectiveness of this therapy on dementia prevention. As a result, it was confirmed that occupation-based reminiscence therapy had a more statistically significant positive effect on subjects' cognitive function, depression level, and quality of life than the conventional conversation-oriented general reminiscence therapy and dementia prevention therapy combined with gymnastic exercise.

To look at this in more detail, first, the occupation-based reminiscence therapy given to the experimental group of this research showed a more significant cognitive function improvement than the control group trained in parallel with the general conversation-oriented general reminiscence therapy and gymnastic exercise. These results imply not only that occupation-based reminiscence therapy for the normal elderly can support significant improvements in cognitive function, in addition to the findings of previous studies that occupation-based reminiscence therapy can help the cognitive function of elderly people with dementia and mild cognitive impairment (MCI) [14-17], as with the results derived from the pilot study of this study, where occupation-based reminiscence therapy was applied to a single group of normal elderly people [22], but also that occupation-based reminiscence therapy can have a more positive effect on improving the cognitive function of normal elderly people compared to the parallel therapy of reminiscence therapy and gymnastic exercise.

In this research, the themes dealt with in the general conversation-oriented reminiscence therapy and task-based reminiscence therapy applied to the control group and experimental group subjects were limited to 8 themes determined through a pre-interview with the subjects focusing on their prior experiences and positive memory of experience. Using these eight themes, a reminiscence therapy activity was conducted for the control group and the experimental group every session for a total of 8 weeks. The findings of this research confirmed a greater cognitive function improvement in the experimental group than in the control group, which means that occupation-based reminiscence therapy, which involves making work along with conversations related to the selected themes of the experimental group, was more effective in improving the cognitive function of elderly people without dementia than the parallel therapy consisting of conversation-oriented reminiscence therapy related to the theme of the control group and a collective gymnastic exercise, regardless of the theme. As mentioned in previous occupation-based reminiscence therapy-related studies [14-17], these outcomes are considered to be the result of occupation-based reminiscence therapy, which includes not only dialogue but also the process of making directly, allowing subjects to use their long-term memory more actively, positively and naturally, while the general reminiscence therapy process in which one's past experience or work know-how related to a theme with a positive experience is shared with other subjects only through dialogue. In addition, the occupation-based reminiscence therapy applied to the experimental group more effectively activated the brain region responsible for detailed procedural memory related to the theme-related making process remaining in the subjects' long-term memory than the parallel therapy applied to the control group. Furthermore, it is the result of interacting with positive emotions in the corresponding long memory and acting more positively on the subjects' cognitive function and improvement of concentration.

In general, it is known that depression level symptoms in the elderly have a negative effect on their quality of life and are closely related to cognitive function [5]. According to previous studies that reported the correlation between depression level symptoms and mild cognitive impairment (MCI) in the elderly, the rate of progression to mild cognitive impairment (MCI) was 1.98 times higher in the elderly with depression level symptoms than in the normal elderly without depression level symptoms [23], and 26 to $63.3 \%$ of the elderly with mild cognitive impairment (MCI) already suffered from depressive symptoms. Furthermore, $20 \%$ of them showed high levels of depressive symptoms [24]. In addition, in previous neuroanatomical studies on depression level symptoms and memory, it has been reported that when depression level symptoms appear in old age, the function of the hippocampus and anterior cortex of the temporal lobe decreases due to neuroanatomical changes in the cerebrum, and as a result, causes the memory of the subject to deteriorate [25]. Based on the results of these previous studies, the depression level symptoms of the elderly can be regarded as an essential element to be managed for effective dementia prevention.

In this research, a relatively significant positive change was observed in the experimental group to which occupation-based reminiscence therapy was applied compared to the control group in terms of subjects' severity of depression level and quality of life. These results imply not only that occupation-based reminiscence therapy for the normal elderly can produce a significant reduction in symptoms of depression level and improvement in cognitive function, in addition to the findings of previous precedent studies that occupation-based reminiscence therapy can help the depression level and cognitive function of elderly people with dementia and mild cognitive impairment (MCI) [14-17], as with the results derived from the pilot study of this study, where occupation-based reminiscence therapy was applied to a single group of normal elderly people [22], but also that occupation-based reminiscence therapy can have a more positive effect on reducing depression level and improving the cognitive function of normal elderly people compared to parallel therapy of reminiscence therapy and gymnastic exercise. 
In general, it is known that the elderly over 65 in Korea suffer from emotional isolation, psychological anxiety such as depression level, and deterioration in overall quality of life due to their physical aging, reduced social activity range, and economic problems. Previous studies related to occupation-based reminiscence therapy have found that subjects participating in occupation-based reminiscence therapy come to have social experiences, such as by playing a kind of teaching role during the therapy process, and through this, self-esteem is improved, emotions are shared, and a sense of belonging that has disappeared after the end of working life is felt [14-17, 26, 27]. It is considered that the experimental group subjects in this research helped each other in the process of using or making tools with other subjects from this stage, when the actual work process was in progress during the course of participating in occupation-based reminiscence therapy, and in the process of making their own creations, obtained a kind of 'teaching role' of informing other subjects of the method, through which their overall self-esteem could be improved. Even in the final stage, the experimental group subjects of this research showed each other their completed work tasks, telling each other not only their various past experiences and emotions at the time, but also their current emotions. Through gaining the opportunity to share positive emotions and express themselves, the subjects were able to experience the process of satisfying social needs. In addition, it seems that they were able to feel a type of sense of belonging through the process of participating in familiar activities with other subjects and achieving a common goal that could be realized together. Finally, as a behavior that was not observed in the control group subjects, it was observed that the experimental group subjects of this research continued to share stories about the program with each other for 2-3 weeks after the program ended. It was also observed that they hoped the program would continue after the end of the overall program. As confirmed in the results of this research, it is believed that the experimental group experienced more of a reduction in depression level and saw positive changes in quality of life compared to the control group through these processes.

The limitation of this research is that only one post-test was performed immediately after therapy in the experimental group and control group was completed, and no evaluation was conducted thereafter, which meant there was a limitation in determining the long-term effect of the occupation-based reminiscence therapy program. In future research, an additional evaluation should be conducted after one week or one month of the intervention process, and the long-term effectiveness of occupation-based reminiscence therapy should also be verified.

\section{Conclusions}

In this research, it was verified that occupation-based reminiscence therapy is a non-pharmacological therapy method that is more effective in improving the cognitive function, reducing symptoms of depression level, and enhancing quality of life of the elderly without dementia in the community com-pared to the conventional parallel therapy of conversation-oriented reminiscence therapy and physical activity. This research is meaningful in that it provides an effective dementia prevention program to the community that can both prevent dementia and help maintain and promote health in the non-demented elderly in the community. Finally, it is expected that this research will be used as an important basis for the establishment and direct implementation of plans for Dementia Prevention Programs for the elderly in various community institutions in the future.

\section{Acknowledgement}

This work was supported by the National Research Foundation of Korea (NRF) grant funded by the Korea government (MSIT) (No.2018R1C1B5086451).

\section{REFERENCES}

[1] T. Hopper et al., "An evidence-based systematic review on cognitive interventions for individuals with dementia," (in eng), Am J Speech Lang Pathol, vol. 22, no. 1, pp. 126-45, Feb 2013, doi: 10.1044/1058-0360(2012/11-0137).

[2] U. Park, "Trends Analysis of Characteristics in Patient with Dementia," Journal of the Korea Academia-Industrial cooperation Society, vol. 18, no. 1, pp. 202-209, 01// 2017. [Online]. Available: https://www.kci.go.kr/kciportal/ci/ser eArticleSearch/ciSereArtiView.kci?sereArticleSearchBean .artiId=ART002194608.

[3] H. Öhman, N. Savikko, T. E. Strandberg, and K. H. Pitkälä, "Effect of physical exercise on cognitive performance in older adults with mild cognitive impairment or dementia: a systematic review," Dement Geriatr Cogn Disord, vol. 38, no. 5-6, pp. 347-65, 2014.

[4] F. Sofi, D. Valecchi, D. Bacci, R. Abbate, G. F. Gensini, A. Casini, and C. Macchi, "Physical activity and risk of cognitive decline: a meta-analysis of prospective studies," J Intern Med, vol. 269, no. 1, pp. 107-17, Jan, 2011.

[5] Y. Lee et al., "Lifestyle Recommendations for Dementia Prevention: PASCAL," J Korean Geriatr Soc, vol. 13, no. 2, pp. 61-68, 6 2009, doi: 10.4235/jkgs.2009.13.2.61.

[6] JS Park, SJ Park, JY Yung, and KG Choi, "Efficacy of Dementia Prevention Program for Cognitive Function, Depression, and Physical Function in the Elderly Non-Demented Women-Focused on Senior Citizens Center," Journal of Korean Society of Occupational Therapy, vol. 23, no. 3, pp. 79-96, 09// 2015. [Online]. Available:https://www.kci.go.kr/kciportal/ci/sereArticleSe arch/ciSereArtiView.kci?sereArticleSearchBean.artiId=AR T002031524. 
[7] J. S. Kwon, "Effects of Cognitive Intervention Activities for the Elderly with Dementia in Korea: A Meta-Analysis," $J$ Health Info Stat, vol. 44, no. 3, pp. 271-278, 8 2019, doi: 10.21032/jhis.2019.44.3.271.

[8] L. Yun, M. Park, and N. Hee, "The Effects of Dementia Prevention Program on Cognition, Depression, Self-esteem and Quality of life in the Elderly with Mild Cognitive Disorder," Korean Journal of Adult Nursing, vol. 19, pp. 787-796, 2007.

[9] EH Yang, "The Effect of Dementia Prevention Program Participation on Cognitive Functions, \& Health Fitness in Elderly Female," Journal of Converging Sport and Exercise Sciences vol. 16, no. 1, pp. 39-52, 2018. [Online]. Available: http://kiss.kstudy.com/thesis/thesis-view.asp?g=kissmeta\& $\mathrm{m}=$ exp\&enc $=\mathrm{D} 14433 \mathrm{DDB} 33 \mathrm{BBFD} 3 \mathrm{BDF} 7 \mathrm{~A} 17428 \mathrm{DED} 19$ 9.

[10] A. Lazar, H. Thompson, and G. Demiris, "A systematic review of the use of technology for reminiscence therapy," (in eng), Health Educ Behav, vol. 41, no. 1 Suppl, pp. 51s-61s, Oct 2014, doi: 10.1177/1090198114537067.

[11] X. D. Peng, C. Q. Huang, L. J. Chen, and Z. C. Lu, "Cognitive behavioural therapy and reminiscence techniques for the treatment of depression in the elderly: a systematic review," (in eng), J Int Med Res, vol. 37, no. 4, pp. 975-82, Jul-Aug 2009, doi: 10.1177/147323000903700 401.

[12] G. Duru Aşiret and S. Kapucu, "The Effect of Reminiscence Therapy on Cognition, Depression, and Activities of Daily Living for Patients With Alzheimer Disease," (in eng), $J$ Geriatr Psychiatry Neurol, vol. 29, no. 1, pp. 31-7, Jan 2016, doi: 10.1177/0891988715598233.

[13] I. Jahanbin, S. Mohammadnejad, and F. Sharif, "The effect of group reminiscence on the cognitive status of elderly people supported by ilam welfare organization in 2013; a randomized controlled clinical trial," (in eng), Int $J$ Community Based Nurs Midwifery, vol. 2, no. 4, pp. 231-9, Oct 2014

[14] YI Choi, "Effects of Occupation Reminiscence Therapy on the Cognitive Function and Self-Esteem in the Elderly with Dementia," Asia-pacific Journal of Multimedia services convergent with Art, Humanities, and Sociology, vol. 9, pp. 781-789, 2019.

[15] T. Nakamae, K. Yotsumoto, E. Tatsumi, and T. Hashimoto, "Effects of Productive Activities with Reminiscence in Occupational Therapy for People with Dementia: A Pilot Randomized Controlled Study*," Hong Kong Journal of Occupational Therapy, vol. 24, no. 1, pp. 13-19, 2014, doi: 10.1016/j.hkjot.2014.01.003.

[16] [16] YJ Cha, "A Study on Occupational Reminiscence Therapy(ORT) Program for the Elderly with Mild Cognitive Impairment(MCI) in Local Community," Journal of the Korea Academia-Industrial cooperation Society, vol. 17, no. 8, pp. 605-614, 08// 2016. [Online]. Available:https://www.kci.go.kr/kciportal/ci/sereArticleSe arch/ciSereArtiView.kci?sereArticleSearchBean.artiId=AR T002142346.

[17] MH Kwon, DH Jung, and SK Kim, "The Effects of Group
Reminiscence Program on Social Interaction and Behavioral Problems in the Resident Elderly With Dementia," The Journal of Occupational Therapy for the Aged and Dementia, vol. 6, no. 2, pp. 11-18, 012012. [Online]. Available: http://scholar.dkyobobook.co.kr/searc hDetail.laf?barcode $=4050025736660$.

[18] J. Y. Lee et al., "Brief screening for mild cognitive impairment in elderly outpatient clinic: validation of the Korean version of the Montreal Cognitive Assessment," (in eng), J Geriatr Psychiatry Neurol, vol. 21, no. 2, pp. 104-10, Jun 2008, doi: 10.1177/0891988708316855.

[19] Z. S. Nasreddine et al., "The Montreal Cognitive Assessment, MoCA: A Brief Screening Tool For Mild Cognitive Impairment," Journal of the American Geriatrics Society, vol. 53, no. 4, pp. 695-699, 2005, doi: https://doi.org/10.1111/j.1532-5415.2005.53221.x.

[20] I. Jung, D. Kwak, S. Joe, and H. S. Lee, "A Study of Standardization of Korean Form of Geriatric Depression Scale(KGDS)," 1997.

[21] H. Lee, D. K. Kim, H. J. Ko, H. M. Ku, E. J. Kwon, and J. H. Kim, "The standardization of geriatric quality of life scale," Korean Journal of Clinical Psychology, vol. 22, pp. 859-881, 01/01 2003.

[22] JH Park, "The Effects of Occupation-based Reminiscence training on Cognition, Depression, and Quality of Life in Community-dwelling Elderly," Journal of Convergence for Information Technology, vol. 11, no. 1, pp. 254-260, 01/31 2021. [Online]. Available:https://www.earticle.net/Article/ A389379.

[23] V. M. Dotson, M. A. Beydoun, and A. B. Zonderman, "Recurrent depressive symptoms and the incidence of dementia and mild cognitive impairment," Neurology, vol. 75 , no. 1, pp. 27-34, 2010, doi: 10.1212/WNL.0b013e3181 e62124.

[24] C. G. Lyketsos, O. Lopez, B. Jones, A. L. Fitzpatrick, J. Breitner, and S. DeKosky, "Prevalence of Neuropsychiatric Symptoms in Dementia and Mild Cognitive ImpairmentResults From the Cardiovascular Health Study," $J A M A$, vol. 288, no. 12, pp. 1475-1483, 2002, doi: 10.1001/jama.288.12.1475.

[25] J. Douglas Bremner, Meena Vythilingam, Eric Vermetten, Viola Vaccarino, and Dennis S. Charney, "Deficits in Hippocampal and Anterior Cingulate Functioning During Verbal Declarative Memory Encoding in Midlife Major Depression," American Journal of Psychiatry, vol. 161, no. 4, pp. 637-645, 2004, doi: 10.1176/appi.ajp.161.4.637.

[26] B. Woods, L. O'Philbin, E. M. Farrell, A. E. Spector, and M. Orrell, "Reminiscence therapy for dementia," (in eng), Cochrane Database Syst Rev, vol. 3, no. 3, p. Cd001120, Mar 1 2018, doi: 10.1002/14651858.CD001120.pub3.

[27] E. J. Lim, "The Relationship between Depression, Cognitive Function and the Instrumental Activities of Daily Living of Elderly Women Living Alone," (in Ko), Journal of the Korea Academia-Industrial cooperation Society, vol. 14, no. 4, pp. 1849-1856, 04/30 2013, doi: 10.5762/kais.2013.14.4.1849. 